\title{
microRNA expression profiling and bioinformatic analysis of dengue virus-infected peripheral blood mononuclear cells
}

\author{
YIMING QI $^{1,2^{*}}$, YING LI $^{1,2^{*}}$, LIN ZHANG $^{1,2^{*}}$ and JUNQI HUANG ${ }^{1,2}$ \\ ${ }^{1}$ Institute of Immunology, Zhongshan School of Medicine, Sun Yat-sen University; \\ ${ }^{2}$ Key Laboratory of Tropical Diseases Control, Ministry of Education, Guangzhou 510080, P.R. China
}

Received August 9, 2012; Accepted January 7, 2013

DOI: $10.3892 / \mathrm{mmr} .2013 .1288$

\begin{abstract}
Dengue virus (DENV) causes self-limiting dengue fever (DF), severe dengue hemorrhagic fever (DHF) and dengue shock syndrome (DSS). It is generally considered that cytokine storm leads to the increased plasma leakage characteristic of DHF/DSS. In the present study, peripheral blood mononuclear cells (PBMCs) were isolated from blood samples of healthy volunteers and infected with DENV serotype 2 (DENV2). Culture supernatants of DENV2-infected and -uninfected PBMCs were analyzed using a human cytokine array. Between a 6-12 h post-infection, levels of CCL5, IL-6 and IL- 8 were markedly elevated, while those of TNF- $\alpha$ decreased. Total RNA isolated from these PBMCs was analyzed by human miRNA microarray to identify differentially expressed microRNAs (miRNAs). Quantitative reverse transcription polymerase chain reaction was used to validate 11 upregulated and 4 downregulated miRNAs. Sanger mibase, miRanda and TargetScan were used to identify 261 common predicted genes. Databases were used to identify homologous sequences on mRNAs of putative target genes that may be directly bound by the miRNAs identified. We found that cytokines and epigenetic regulators may be putative target genes of these miRNAs. Using ingenuity pathway analysis, we noted that canonical pathways, including biological regulation, may be modulated by these miRNAs.
\end{abstract}

Correspondence to: Dr Junqi Huang, Institute of Immunology, Zhongshan School of Medicine, Sun Yat-sen University and Key Laboratory of Tropical Diseases Control, Ministry of Education, Guangzhou 510080, P.R. China

E-mail: huangjunqi1410@163.com

*Contributed equally

Abbreviations: DENV, dengue virus; DF, dengue fever; DHF, dengue hemorrhagic fever; DSS, dengue shock syndrome; IPA, ingenuity pathway analysis; m.o.i., multiplicity of infection; miRNAs, microRNAs; PBMCs, peripheral blood mononuclear cells

Key words: dengue virus, peripheral blood mononuclear cells, microRNAs, bioinformatic analysis

\section{Introduction}

Dengue virus (DENV), with 4 serologic types, is one of the most prevalent flaviviruses to have re-emerged during recent decades. The virus has been identified globally and is no longer associated with tropical and subtropical countries only. Due to lack of vaccines and specific treatments, $\sim 2.5$ billion individuals from $>110$ countries are at risk of DENV infection each year and $>500,000$ individuals are affected by the potentially fatal dengue hemorrhagic fever (DHF) or dengue shock syndrome (DSS) (1). Therefore, studies on non-vaccine surrogates to control the spread of DENV and eradicate severe forms of the infection are urgently required.

Although the pathogenesis of DHF/DSS remains to be adequately elucidated, immune enhancement, cytokine storm, secondary infection and virulence variation all appear to contribute to the devastating physiopathology of DENV infection. It is hypothesized that a number of clinical complications are closely associated with the host immune response $(2,3)$. In specific cases of secondary infection, a burst of cytokines and additional inflammatory molecules from cross-reactive $\mathrm{T}$ cells, mast or endothelial cells has been observed (4). A complex interactive network, that includes synergistic and antagonistic effects of the initial cytokines, in turn induces a cascade of additional cytokines and inflammatory mediators (4).

The expression of cytokine encoding genes is regulated and affected by a number of endogenous and exogenous factors. Recently, microRNAs (miRNAs) were identified as key components of immune regulation (5). Mature miRNAs, 22-25 nucleotides in length, downregulate mRNA expression through the assembly of a miRNA-induced silencing complex known as RISC, which binds complementary sequences in target mRNAs and subsequently causes catalytic cleavage or transcriptional repression of target mRNAs (6). Several miRNAs were demonstrated to regulate downstream inflammatory responses by directly targeting genes encoding molecules that are secreted by or are expressed on the surface of immune cells (7-9). Specific miRNAs are capable of targeting genes that control epigenetic pathways. These miRNAs affect the expression of certain epigenetic regulators, including histone deacetylases, DNA methyltransferases and polycomb group genes. This network between miRNAs and epigenetic pathways appears to form an epigenetic-miRNA regulatory circuit and regulate genome expression (10). 
In the present study, we hypothesized that, following DENV infection, excessive secretion of cytokines and inflammatory mediators occurs, in part, as a result of the activity of miRNAs that target the corresponding genes or regulators. Using a cytokine array and miRNA microarray, we examined the expression of inflammatory cytokines and profile of miRNAs, respectively, in DENV serotype 2 (DENV2)-infected peripheral blood mononuclear cells (PBMCs). Data analysis was performed using several online databases to elucidate the potential molecular mechanisms underlying the pathology of DENV infection. This in-depth study provides novel insight into potential therapeutic treatments to attenuate the vigorous host immune responses in DENV infection.

\section{Materials and methods}

Collection of blood samples from donors. All blood samples and procedures in this study were approved by the Ethics Committee of Sun Yat sen University. Healthy volunteers provided voluntary informed consent. Blood samples were provided by healthy volunteers $(n=6)$ at the Zhongshan School of Medicine (Sun Yat-sen University, China). Samples were processed independently. PBMCs were isolated from each sample by Ficoll-Hypaque density gradient centrifugation. PBMCs were then washed once in RPMI-1640 medium and resuspended in the same medium containing $10 \%$ inactivated fetal calf serum. PBMCs were cultured in 24-well culture plates (all obtained from Gibco, Carlsbad, CA, USA) and incubated at $37^{\circ} \mathrm{C}$ in $5 \% \mathrm{CO}_{2}$ for 6 or $12 \mathrm{~h}$.

Expansion and titration of DENV2. The DENV2 serotype used in this study originated from the New Guinea C strain. The virus was bred in C6/36 mosquito cells that were cultured in Eagle's Minimal Essential Medium containing 10\% fetal bovine serum (FBS). The viral suspension was added to the cell cultures and incubated for 3-5 days. The virus was harvested from the culture supernatant by centrifugation to separate the cells and cell debris. The supernatant was stored at $-80^{\circ} \mathrm{C}$ until required. To determine the plaque-forming units (PFU) of the viral suspension, C6/36 cells were first seeded in a 24-well plate at a concentration of $1 \times 10^{6}$ cells/well and then infected with serial dilutions of the viral suspension. The plate was incubated at $37^{\circ} \mathrm{C}$ for 5 days. Plaques were visualized by staining with $0.5 \%$ crystal violet and $\mathrm{PFU} / \mathrm{ml}$ was estimated using a previously described method (11).

Infection of PBMCs with DENV2. Six of the PBMC cultures obtained from blood samples of the healthy volunteers were used for DENV2 infection. Confluent cultures of PBMCs in 24-well plates were rinsed with RPMI-1640 medium and infected with the viral suspension in the same medium, at a multiplicity of infection of $4 \mathrm{PFU} / 10^{6}$ cells. Cells were infected for $2 \mathrm{~h}$ at $37^{\circ} \mathrm{C}$. Following cell infection, the medium was discarded and replaced with RPMI-1640 medium containing $4 \%$ FBS and the plates were incubated further for 6 or $12 \mathrm{~h}$. Culture supernatants containing DENV2 were separated from cells and cell debris by centrifugation at $1,000 \mathrm{x} \mathrm{g}$.

Cytokine protein array. Culture supernatants of DENV2infected and -uninfected PBMCs were collected, as described, at 0,6 and $12 \mathrm{~h}$ post-DENV2 infection. Equal volumes $(1 \mathrm{ml})$ of each culture supernatant were incubated with the precoated Proteome Profiler array membrane (ARY005, Human Cytokine Antibody Array kit; R\&D Systems, Minneapolis, MN, USA) and processed according to the manufacturer's instructions. Densitometric analysis of dot blots was performed using Image J software (National Institutes of Health, Bethesda, MD, USA), as described previously (12).

Total RNA extraction. Total RNA was isolated from cultured DENV2-infected and -uninfected (both $\mathrm{n}=6$ ) PBMCs using TRIzol (Invitrogen, Carlsbad, CA, USA) and miRNeasy mini kit (Qiagen, Dusseldorf, Germany) according to the manufacturer's instructions. RNA quality and quantity was measured using a Nanodrop spectrophotometer (ND-1000; Nanodrop Technologies, Wilmington, DE, USA). RNA integrity was confirmed by gel electrophoresis.

miRNA microarray analysis. RNA samples were labeled using the miRCURY ${ }^{\mathrm{TM}} \mathrm{Hy}^{\mathrm{TM}} / \mathrm{Hy}^{\mathrm{TM}}$ Power labeling kit according to the manufacturer's instructions. The $\mathrm{Hy}^{\mathrm{TM}}{ }^{\mathrm{TM}}$-labeled samples were hybridized to the miRCURY Tм LNA Array (v.16.0) according to the manufacturer's instructions. Following hybridization, the slides were washed 3 times using wash buffer from the kit and dried by centrifugation for $5 \mathrm{~min}$ at 1,000 $\mathrm{xg}$ (all obtained from Exiqon, Vedbaek, Denmark). Slides were scanned using the Axon GenePix 4000B microarray scanner (Axon Instruments, Inc., Foster City, CA, USA). Scanned images were imported into GenePix Pro 6.0 software for grid alignment and data extraction. Replicate miRNA in duplicate samples was averaged and miRNAs with intensities of $\geq 50$ in all samples from each group (DENV2-infected or control groups) were selected for calculation of the normalization factor. Expressed data were median-normalized. Following normalization, significantly differentially expressed miRNAs were identified through Volcano Plot filtering. Hierarchical clustering was performed using MEV software (v4.6; TIGR, http://compbio.dfci.harvard.edu/tgi/software/).

Quantitative reverse transcription polymerase chain reaction analysis (qRT-PCR) of miRNAs. Microarray data on differential expression were verified for selected genes by qRT-PCR using extracted total RNA samples as templates. Primers were synthesized at Life Technologies (Life Technologies, Grand Island, NY, USA). Gene Amp PCR System 9700 (Applied Biosystems, Foster City, CA, USA) was used to synthesize cDNAs for RT-PCR. RT-PCR amplification was performed on the ABI PRISM 7900 System (Applied Biosystems). Total RNA from the donors used for the miRNA microarrays was used to assay hsa-let-7e, hsa-miR-4290, $-4279,-451$, $-720,-491-3 \mathrm{P},-451,-4286,-3653,-30 \mathrm{~b},-20 \mathrm{a},-106 \mathrm{~b},-222$, $-320 \mathrm{c},-767-5 \mathrm{p},-1246,-33 \mathrm{a},-1290,-3686,-625^{*}$, by qRT-PCR (Table I). Briefly, 500 ng total RNA was reverse-transcribed to cDNA $\left(60^{\circ} \mathrm{C}\right.$ for $5 \mathrm{~min}, 37^{\circ} \mathrm{C}$ for $\left.60 \mathrm{~min}\right)$ using MMLV reverse transcriptase (Epicentre Biotechnologies, Madison, WI, USA). For quantification of each miRNA, a quantitative PCR step (cycle program: denaturation for $10 \mathrm{~min}$ at $95^{\circ} \mathrm{C}$ and 40 cycles of denaturation, $10 \mathrm{sec}$ at $95^{\circ} \mathrm{C}$ and amplification, $1 \mathrm{~min}$ at $60^{\circ} \mathrm{C}$ ) was performed on the Takara PCR Thermal Cycler (Takara Bio, Inc., Shiga, Japan). RT-PCR 
Table I. qRT-PCR and RT-PCR primers.

\begin{tabular}{|c|c|c|c|}
\hline Experiment & Gene name & Direction & Primer sequence \\
\hline \multirow[t]{38}{*}{ qRT-PCR } & snRNA U6 & $\mathrm{F}$ & 5'-CCCTGCGCAAGGATGAC-3' \\
\hline & & $\mathrm{R}$ & 5'-GTCGGTGTCGTGGAGTCG-3' \\
\hline & hsa-miR-4290 & GSP & 5'-TGCCCTCCTTTCTTCCC-3' \\
\hline & & $\mathrm{R}$ & 5'-GTCGGTGTCGTGGAGTCG-3' \\
\hline & hsa-miR-4279 & GSP & 5'-GCTCTCCTCCCGGCTT-3' \\
\hline & & $\mathrm{R}$ & 5'-GTCGGTGTCGTGGAGTCG-3' \\
\hline & hsa-miR-720 & GSP & 5'-GGTTCTCGCTGGGGC-3' \\
\hline & & $\mathrm{R}$ & 5'-GTCGGTGTCGTGGAGTCG-3' \\
\hline & hsa-miR-491-3p & GSP & 5'-GGCTTATGCAAGATTCCCT-3' \\
\hline & & $\mathrm{R}$ & 5'-GTCGGTGTCGTGGAGTCG-3' \\
\hline & hsa-miR-451 & GSP & 5'-GGAAACCGTTACCATTACTGAG-3' \\
\hline & & $\mathrm{R}$ & 5'-GTCGGTGTCGTGGAGTCG-3' \\
\hline & hsa-miR-4286 & GSP & 5'-GGGACCCCACTCCTG-3' \\
\hline & & $\mathrm{R}$ & 5'-GTCGGTGTCGTGGAGTCG-3' \\
\hline & hsa-miR-3653 & GSP & 5'-GGGGGCTAAGAAGTTGACT-3' \\
\hline & & $\mathrm{R}$ & 5'-GTCGGTGTCGTGGAGTCG-3' \\
\hline & hsa-miR-30b & GSP & 5'-GGGTGTAAACATCCTACACTCA-3' \\
\hline & & $\mathrm{R}$ & 5'-GTCGGTGTCGTGGAGTCG-3' \\
\hline & hsa-miR-20a & GSP & 5'-GGGGTAAAGTGCTTATAGTGC-3' \\
\hline & & $\mathrm{R}$ & 5'-GTCGGTGTCGTGGAGTCG-3' \\
\hline & hsa-miR-106b & GSP & 5'-GGGGTAAAGTGCTGACAGTG-3' \\
\hline & & $\mathrm{R}$ & 5'-GTCGGTGTCGTGGAGTCG-3' \\
\hline & hsa-miR-320c & GSP & 5'-GGAAAAGCTGGGTTGAGAG-3' \\
\hline & & $\mathrm{R}$ & 5'-GTCGGTGTCGTGGAGTCG-3' \\
\hline & hsa-miR-767-5p & GSP & 5'-TGCACCATGGTTGTCTGAG-3' \\
\hline & & $\mathrm{R}$ & 5'-GTCGGTGTCGTGGAGTCG-3' \\
\hline & hsa-miR-1246 & GSP & 5'-GGGAATGGATTTTTGGAGC-3' \\
\hline & & $\mathrm{R}$ & 5'-GTCGGTGTCGTGGAGTCG-3' \\
\hline & hsa-miR-33a & GSP & 5'-GGGGTGCATTGTAGTTGC-3' \\
\hline & & $\mathrm{R}$ & 5'-GTCGGTGTCGTGGAGTCG-3' \\
\hline & hsa-miR-1290 & GSP & 5'-GGGGTGGATTTTTGGATC-3' \\
\hline & & $\mathrm{R}$ & 5'-GTCGGTGTCGTGGAGTCG-3' \\
\hline & hsa-miR-3686 & GSP & 5'-GGGGATCTGTAAGAGAAAGTAAAT-3' \\
\hline & & $\mathrm{R}$ & 5'-GTCGGTGTCGTGGAGTCG-3' \\
\hline & hsa-let-7e & GSP & 5'-GGGTGAGGTAGGAGGTTGTAT-3' \\
\hline & & $\mathrm{R}$ & 5'-GTCGGTGTCGTGGAGTCG-3' \\
\hline & hsa-miR- $625^{*}$ & GSP & 5'-CCGACTATAGAACTTTCCCC-3' \\
\hline & & $\mathrm{R}$ & 5'-GTCGGTGTCGTGGAGTCG-3' \\
\hline \multirow[t]{3}{*}{ RT-PCR } & snRNA U6 & - & 5'-GTCGGTGTCGTGGAGTCG-3' \\
\hline & miR-RT & - & 5'-GTCGGTGTCGTGGAGTCGTTTGCAATTGC \\
\hline & & & ACTGGATTTTTTTTTTTTTTTTTTV-3 \\
\hline
\end{tabular}

GSP, gene-specific primer; R, reverse primer. miR, microRNA; qRT-PCR, quantitative reverse transcription polymerase chain reaction; snRNA, small nuclear RNA.

was performed using the sample mi-RNA (miR-RT) and the internal reference was non-coding small nuclear RNA (snRNA U6) as previously described (13).
Bioinformatics analysis. To predict target genes of highly differentially expressed miRNAs by correlating their abundance with expression of cytokine genes, the correlation of 
Table II. Comparison of miRNA expression in DENV2-infected and control PBMCs.

\begin{tabular}{|c|c|c|c|}
\hline miRNA & Chromosomal location & Fold change (I/C) & Parametric P-value \\
\hline hsa-miR-4290 & 9 & 36.627 & $2.85 \mathrm{E}-02$ \\
\hline hsa-miR-4279 & 5 & 21.815 & $1.29 \mathrm{E}-02$ \\
\hline hsa-miR-625 & $14 \mathrm{q} 23.3$ & 8.987 & $3.77 \mathrm{E}-02$ \\
\hline hsa-let-7e & $19 q 13.41$ & 8.585 & $2.53 \mathrm{E}-02$ \\
\hline hsa-miR-3686 & 8 & 7.728 & $4.63 \mathrm{E}-03$ \\
\hline hsa-miR-1200 & 1 & 6.929 & $1.62 \mathrm{E}-03$ \\
\hline hsa-miR-33a & $22 q 13.2$ & 6.244 & $1.16 \mathrm{E}-02$ \\
\hline hsa-miR-378a & $5 q 32$ & 4.773 & $2.90 \mathrm{E}-02$ \\
\hline hsa-miR-1246 & $2 q 31.1$ & 3.295 & 7.33E-03 \\
\hline hsa-miR-767-5p & $\mathrm{Xq} 28$ & 3.184 & $1.67 \mathrm{E}-02$ \\
\hline hsa-miR-320c & 18Q11.2 & 3.014 & 2.33E-02 \\
\hline hsa-miR-720 & 3 & 2.038 & $1.21 \mathrm{E}-02$ \\
\hline hsa-miR-491-3p & $9 q 21.3$ & 1.98 & $1.15 \mathrm{E}-02$ \\
\hline hsa-miR-451 & $17 \mathrm{q} 11.2$ & 1.918 & 4.32E-02 \\
\hline hsa-miR-4286 & 8 & 1.861 & 4.63E-03 \\
\hline hsa-miR-106b & $7 q 22.1$ & 0.411 & $5.00 \mathrm{E}-03$ \\
\hline hsa-miR-20a & $13 \mathrm{q} 31.3$ & 0.494 & $6.14 \mathrm{E}-04$ \\
\hline hsa-miR-30b & $8 \mathrm{q} 24.22$ & 0.542 & $1.51 \mathrm{E}-02$ \\
\hline hsa-miR-3653 & 22 & 0.603 & $3.56 \mathrm{E}-03$ \\
\hline
\end{tabular}

Significantly differentially expressed miRNAs in DENV2-infected PBMCs (I) relative to uninfected control (C) PBMCs, as estimated by microarray analysis $(\mathrm{P}<0.05)$. PBMC miRNA expression is presented as the fold change $(\mathrm{I} / \mathrm{C})$. DENV2, dengue virus serotype 2 ; PBMCs, peripheral blood mononuclear cells; miRNA/miR, micro RNA.

miRNA and target genes was examined using three web-based databases: Sanger mibase (http://www.mirbase.org/), miRanda (http://www.microrna.org/microrna/home.do/) and TargetScan (http://www.targetscan.org/). Target genes were overlapped with sequences from each database to identify common predicted genes and develop a mechanistic understanding of cytokine secretion in PBMCs following DENV infection. To analyze the pathways that these targets may collectively regulate, ingenuity pathway analysis (IPA) software was used to identify specific pathways predicted to be modulated by these miRNAs. $\mathrm{P}<0.05$ was considered statistically significant.

\section{Results}

Identification of differentially expressed cytokines during DENV2 infection in PBMCs. The excessive release of cytokines following DENV infection is hypothesized to be responsible for enhanced vascular permeability that results in fatal plasma leakage and shock in DHF and DSS. Therefore, a human cytokine array was performed to identify the differentially expressed cytokines in DENV-infected PBMCs. PBMCs were isolated from blood samples of healthy volunteers and cultured in vitro. Supernatants were collected at 0 , 6 and $12 \mathrm{~h}$ following DENV2-infection. Protein extracts from culture supernatants were allowed to react with a cocktail of anti-cytokine antibodies and subsequently incubated with the array of capture antibodies that detect 36 cytokines. A large immune response was evident in the infected PBMCs compared with control, from the capture of cytokines on the array (Fig. 1A). Overexpressed cytokines included: CCL5 and 3 , PAI-1 and IL-6, -8, $-1 \alpha$ and $-1 \beta$. Downregulated cytokines included: TNF- $\alpha$, CCL4, MCP-1 and IL-10. Based on the results of the cytokine array, CCL5 and IL-6 and -8 were the most highly expressed immunomodulators (Fig. 1C), expressed at least four times higher in DENV2-infected PBMCs than in DENV2-uninfected cells.

Differential miRNA expression between DENV2-infected and -uninfected PBMCs. To test our hypothesis that miRNAs are involved in gene expression changes during DENV infection, total RNA was extracted from the DENV2-infected and control PBMCs and analyzed by microarray (Fig. 2A). miRNAs that were differentially expressed during DENV2 infection were identified (Table II). Among these, upregulated miRNA subsets included: miR-4290, $-4279,-625^{*}$, -let-7e, $-1290,-33 a$, $-378,-1246,-767-5 p,-320 c,-720,-491-3 p,-3647,-451$ and -4286. Downregulated miRNA subsets included: miR-106b, $-20 \mathrm{a},-30 \mathrm{~b}$ and -3653 (Table II). miRNAs that exhibited a fold change $(\mathrm{FC})>1.5$ and a P-value $<0.05$ for the comparison of levels in infected and control RNAs were analyzed further by qRT-PCR (Fig. 2B).

Authentication of the miRNA signatures by $q R T-P C R$. To confirm differential expression of the miRNAs identified in 
Table III. Alignment of sequences from mRNA of target genes encoding cytokines with miRNA sequences that were differentially expressed in DENV2-infected compared with -uninfected PBMCs.

Sequence in target mRNA/miRNA

Matching sequence

IL-6 3' UTR/hsa-let-7e

5'-UGGAAAGUGUAGGCUUACCUCAA-3'

CCL3 3' UTR/hsa-let-7e

3'-UUGAUAUGUUGGAGGAUGGAGU-5'

5'-GUGUGACCUCCACAGCUACCUCU-3'

3'-UUGAUAUGUUGGAGGAUGGAGU-5'

CXCL1 3' UTR/hsa-miR-4279

5'-GUUUGAGCAUCGCUUAGGAGAAG-3'

3'-CUUCGGCCCUCCUCUC-5'

MIF 3' UTR/hsa-miR-451

5'-UGGUGGGGAGAAAUAAACGGUUU-3'

3'-UUGAGUCAUUACCAUUGCCAAA-5'

CCL5 3' UTR/hsa-miR-106b

5'-GCCUGUAAUCCCAGCACUUUG-3'

3'-GACGUGACAGUCGUGAAAU-5'

EZH2 3' UTR/hsa-let-7e

5'-NNNNNNNNNNCAUCUGCUACCUCC-3'

3'-UUGAUAUGUUGGAGGAUGGAGU-5'

DNMT3A 3' UTR/hsa-miR-30b

5'-GUACAGGGCUUCACAGUUUACAA-3'

3'-UCGACUCACAUCCUACAAAUGU-5'

DENV2, dengue virus serotype 2; miRNA/miR, micro RNA; PBMCs, peripheral blood mononuclear cells; UTR, untranslated region.

A

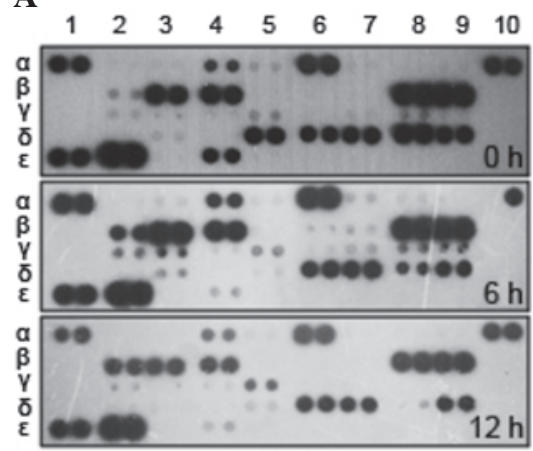

B

\begin{tabular}{|c|c|c|c|c|c|c|c|c|c|c|}
\hline & 1 & 2 & 3 & 4 & 5 & 6 & 7 & 8 & 9 & 10 \\
\hline & & C5a & CD154 & G-CSF & GM-CSF & CXCL & CCL1 & CD54 & IFN-y & Pos. \\
\hline & & IL-1a & IL-1 $\beta$ & IL-1RA & IL-2 & IL-4 & IL-5 & IL-6 & IL-8 & \\
\hline & & IL-10 & |L-12p70 & IL-13 & IL-16 & IL-17 & |LL-17E & IL-23 & IL-27 & \\
\hline б & & IL-32a & IP-10 & I-TAC & MCP-1 & MIF & CCL3 & CCL4 & PAl-1 & \\
\hline & os. & CCL5 & CXCL12 & TNF- $a$ & sTREM & & & & & $\mathrm{Neg}$ \\
\hline
\end{tabular}

C

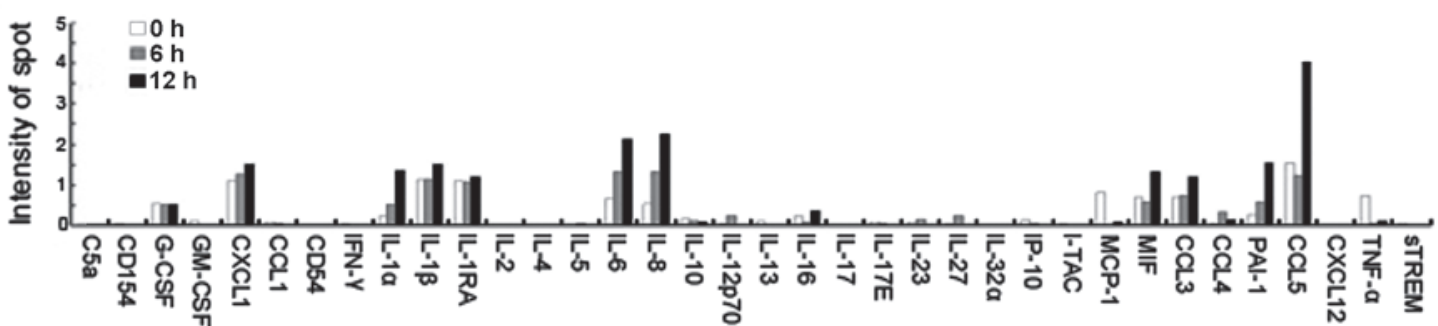

Figure 1. Inflammatory response in PBMCs during DENV2 infection. PBMCs were isolated from blood samples collected from healthy donors and infected with DENV2 for 6 or $12 \mathrm{~h}$ (0 h as uninfected control). Protein was extracted from PBMC culture supernatants and analyzed by cytokine microarray. Signal intensities between DENV2-infected and -uninfected samples were compared to identify differentially expressed cytokines in DENV2 infection. (A) Relative cytokine expression following 0, 6 and 12 h of DENV2 infection. Array images were captured following 5-min exposure to X-ray film. (B) Template demonstrating the location of spots of Pos and Neg controls and various cytokine capture antibodies. All spots were performed in duplicate. (C) Histogram presenting spot intensities (arbitrary units) indicative of levels of cytokines at 0, 6 or $12 \mathrm{~h}$ in DENV2-infected PBMCs. DENV2, dengue virus serotype 2; PBMCs, peripheral blood mononuclear cells; Pos, positive; Neg, negative.

the microarray described, qRT-PCR analysis was performed on the RNA samples isolated from DENV2-infected or -uninfected PBMCs. qRT-PCR results were normalized using an internal control (snRNA U6) to reduce sample variation (data not shown). Expression of miR-4290, -1290 and-33a and let-7e revealed significant upregulation with a high $\mathrm{FC}$ ranging between 7.92 and 14.87. By contrast, the downregulation of miR-106b, -20a and -30b was identified with FCs ranging between 0.41 and 0.60 (Fig. 2B).

Target gene and pathway prediction by bioinformatic analysis. The function of miRNAs that were highly differentially 

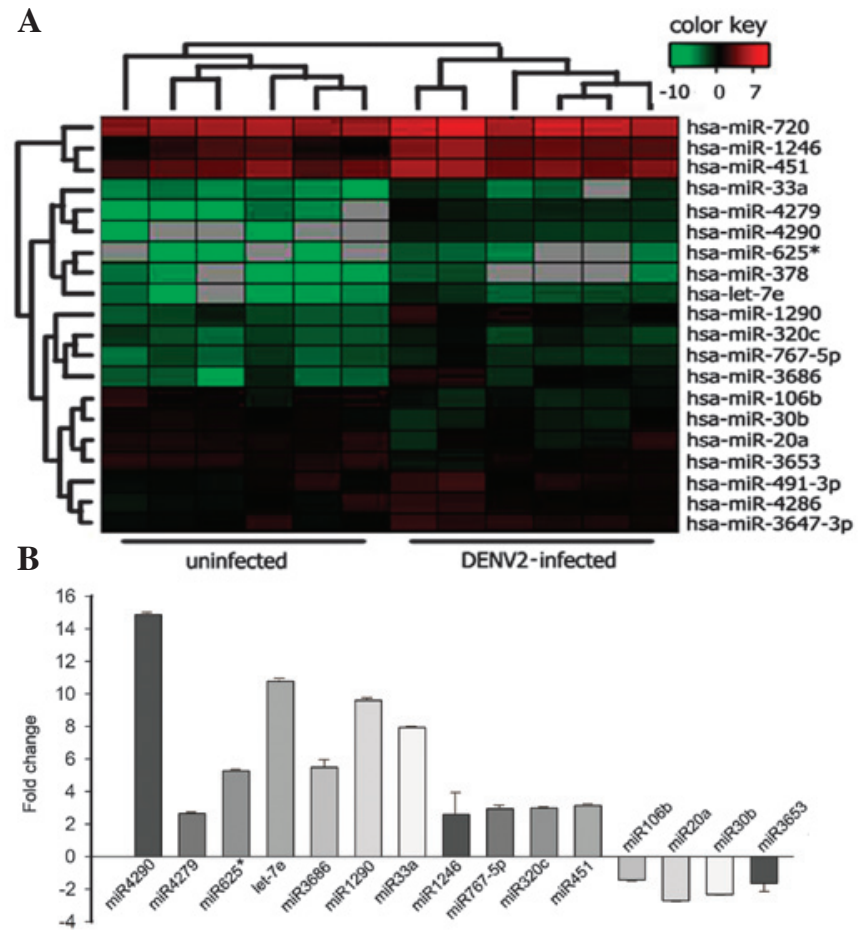

Figure 2. miRNA expression in PBMCs following infection with DENV2. Levels of various miRNAs in DENV2-infected and control PBMCs was compared by microarray analysis. $\mathrm{P}<0.05$ was considered to indicate miRNAs that were significantly differentially expressed. (A) Hierarchical cluster analysis of PBMCs infected or uninfected by DENV2 is presented following filtering. Range of fold change is between -10 and 7. Red, green and grey boxes indicate upregulated, downregulated and undetected genes, respectively. (B) miRNA expression levels in PBMCs were analyzed by multiplex qRT-PCR. Numbers represent the fold change of $\mathrm{Ct}$ values $(\Delta \mathrm{Ct})$ between DENV2-infected and -uninfected groups following normalization to an internal control (snRNA U6). DENV2, dengue virus serotype 2; PBMCs, peripheral blood mononuclear cells; miRNA/miR, micro RNA; qRT-PCR, quantitative reverse transcription polymerase chain reaction; snRNA, small nuclear RNA.

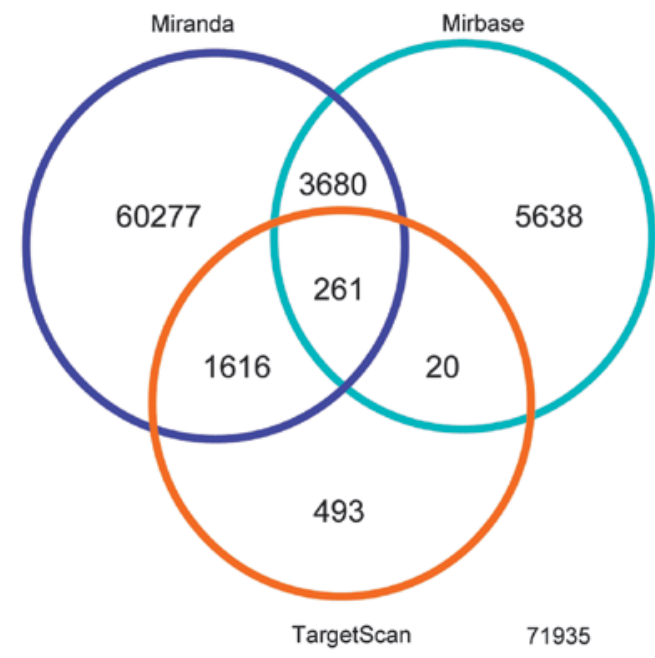

Figure 3. Target gene prediction by 3 miRNA databases, Sanger mibase, miRanda and TargetScan. miRNA, micro RNA.

expressed between DENV2-infected and -uninfected PBMCs was analyzed. The online databases of human miRNAs, Sanger mibase, miRanda and TargetScan v4.2 were used to

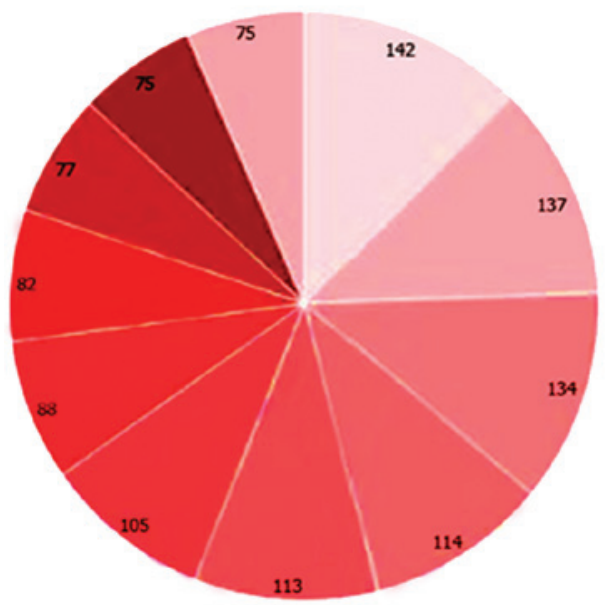

biological regulation
n regulation of biological process
n macromolecule metabolic process
- response of stimulus
- cellular macromolecule metabolic process
- cellular response of stimulus
- cellular metabolic process
- regulation of metabolic process
- signal transduction

Figure 4. Online databases of human miRNAs and IPA software were used to predict genes that may be targeted by the miRNAs found to be highly differentially expressed during DENV2 infection in PBMCs. The pie chart presents the number of putative target genes belonging to various gene families classified on the basis of their involvement in the various cell processes. DENV2, dengue virus serotype 2; miRNA, micro RNA; IPA, ingenuity pathway analysis; PBMCs, peripheral blood mononuclear cells.

predict target groups of the differentially expressed miRNAs by correlating their abundance with the expression of cytokine genes. When targets were overlapped with each database, 261 common predicted genes were detected (Fig. 3). Combined targets were submitted to computational analysis to identify pathways that may be collectively regulated. Using IPA software, canonical pathways, including pathways associated with biological regulation, cell response to stimulus, signal transduction and metabolism were predicted to be markedly modulated by these miRNAs (Fig. 4).

Cytokine storm-related miRNAs and epigenetic regulators. To examine whether following DENV2 infection, differential expression of miRNAs is associated with elevated levels of cytokines, we performed specific sequence homology searches to identify a match between the sequences of chemokine-encoding genes and miRNAs that were differentially expressed during DENV2-infection (Fig. 2B). Of note, the $5^{\prime}$ region of the miR-let-7e (low expression) had a sequence that was complementary to the 3 '-untranslated region (UTR) elements of IL-6 and CCL3. Similarly, mRNA corresponding 
to MIF and CCL5 appeared to be targeted by miR-451 and $-106 \mathrm{~b}$, respectively. In addition, CXCL1 was revealed as a potential target of miR-4279 that was differentially expressed during DENV2-infection (Table III). The miR-let-7e sequence was also found to be complementary to the target sequence of EZH2 mRNA, a conserved catalytic subunit within PRC2 that initiates silencing by catalyzing histone H3 Lysine 27 methylation in an initial step. The miR-30b sequence was complementary to the target sequence in the mRNA of DNA methyltransferase 3A (Table III).

\section{Discussion}

Currently, it is hypothesized that excessive release of pro-inflammatory molecules, largely by $\mathrm{T}$ cells and mast and endothelial cells, is responsible for damage to endothelial cells in DHF and DSS. Cytokines, rather than viruses, bind to vascular endothelial cells directly, resulting in pleiotropic effects which include enhanced inflammation and induction of vascular leakage (4). Although cytokine storm is primarily attributed to massive T-cell activation following secondary DENV infection, a number of studies indicate that innate immune response-related cytokines are also involved, particularly in severe cases of primary dengue infection (14). In particular, high concentrations of cytokines, including IFN- $\gamma$, TNF- $\alpha$ and IL-10, in the serum of patients with severe DENV infections have been widely reported in Vietnam (15), India (16) and Cuba (17), as well as elevated levels of IL-6 in children with ascites (18). Additional studies have demonstrated that endothelial cells induce a high level of immune cell-mediated recruitment of cytokines IL-6/8, CXCL9/10/11, CCL5 and IL-7 (19). In the present study, levels of specific immunomodulators in DENV2-infected PBMCs were elevated, including CCL5 and IL-6 and -8, while those of TNF- $\alpha$, IL-10, MCP-1 and CCL4 were decreased compared with uninfected PBMCs. In this study, PBMCs were isolated from blood samples of healthy donors with no history of DENV infection and PBMC cultures were infected with DENV2 in vitro. This procedure avoided any interference or variability in samples that may be introduced by secondary infections or differential severity of infection.

Mature miRNAs are small, non-coding RNAs that are largely identified in mammalian cells, functioning in a similar manner to small interfering RNAs. Single-stranded miRNAs bind to the 3'-UTR of target mRNAs through partial sequence homology and induce translation blockage or, less frequently, mRNA degradation (20). A previous study indicated that miRNAs are able to silence gene expression primarily by destabilizing target mRNA, thereby reducing their abundance (21). In addition to functioning as tumor suppressor genes, miRNAs are widely recognized to be associated with physiological processes, including growth, differentiation, apoptosis, stress responses and T-cell homeostasis, as well as pathological processes, including initiation, progression and prognosis of numerous diseases (22).

miRNAs were previously reported to be closely associated with pathogenesis of flaviviruses. miR-124a, -128a, -218 and -let-7c may be important for neurological symptoms caused by a chimeric tick-borne encephalitis/dengue virus (23). Additional studies have demonstrated that miR-122 (24) and
$-142(25)$ of the host cells are involved in restricting dengue virus replication. Tolfvenstam et al identified high levels of secretion of cytokines, including MCP-1, CCL8, IP-10 and CCL3, in dengue-infected peripheral blood cells, hypothesizing that these cytokines may be negatively regulated by miR-147 (26). In the present study, miR-106b, -20a and $-30 b$ were observed to be downregulated during DENV2 infection with an FC ranging between 0.41 and 0.60 . We hypothesized that decreased miRNAs may relieve inhibition of target genes, leading to the observed increase in levels of pro-inflammatory cytokines. In support of this hypothesis, bioinformatic analyses reveal that abundance of miR-106b, which may bind CCL5 mRNA, was decreased during DENV2 infection. Therefore, it is likely that during DENV2 infection, decreased miR-106b may lead to derepression of CCL5 expression and enhance secretion of this cytokine.

In contrast to miR-106b, other miRNAs, including miR-4290, -let-7e, -1290 and-33a were highly expressed during DENV2-infection-induced cyctokine activation. Of these, miR-let-7e may bind CCL3 and IL-6 mRNA, levels of which are also elevated during DENV2 infection. As discussed, miRNAs may regulate gene expression not only by destabilizing mRNAs by direct binding, but also via epigenetic mechanisms (10). Transcription initiation may be regulated via epigenetic processes that are dependent on chromatin structure. Open and accessible chromatin (euchromatin) facilitates active and selective gene transcription, whereas closed and condensed chromatin (heterochromatin) is unable to be translated into mRNA. These chromatin structures are markedly affected by DNA-binding proteins, including histones and chromatin remodeling processes i.e., histone modifications and DNA methylation. It is known that miRNAs target specific epigenetic regulatory proteins, including EZH2. Therefore, we propose that epigenetic modulation may account for differential miRNA expression during the DENV cytokine storm.

In the present study, molecular changes in PBMCs post-DENV2 infection were analyzed by microarray and cytokine array. A number of cytokines were identified to be abundantly secreted and differentially expressed miRNAs were revealed between DENV2-infected and control samples. In addition, putative targets of specific differentially expressed miRNAs were identified using bioinformatics analysis. Based on previous studies and data presented in the current study, specific differentially expressed miRNA appear to cause de-repression of cytokine expression, while additional miRNAs target epigenetic modulators of cytokine expression. Further investigations should be performed to determine molecules that interact with these miRNAs and elucidate the molecular mechanisms that trigger cytokine storm, a major pathophysiological event following DENV infection.

\section{Acknowledgements}

The present study was supported by National Natural Science Foundation of China (no. 30872350), Natural Science Foundation of Guangdong Province (nos. 81510008901000017 and S2012010009050), Guangdong Province Scientific Technology Project (nos. 2010B050700008 and 2011B040300022), Guangzhou City Scientific Technology 
Project (nos. 2011J4100084 and 2008Z1-E221) and The Fundamental Research Funds for the Central Universities (no. 10YKPY31). The authors thank additional members of the laboratory for their valuable comments.

\section{References}

1. Guzman MG and Vazquez S: The complexity of antibody-dependent enhancement of dengue virus infection. Viruses 2: 2649-2662, 2010.

2. Martina BE, Koraka P and Osterhaus AD: Dengue virus pathogenesis: an integrated view. Clin Microbiol Rev 22: 564-581, 2009

3. Green S and Rothman A: Immunopathological mechanisms in dengue and dengue hemorrhagic fever. Curr Opin Infect Dis 19: 429-436, 2006.

4. Pang T, Cardosa MJ and Guzman MG: Of cascades and perfect storms: the immunopathogenesis of dengue haemorrhagic fever-dengue shock syndrome (DHF/DSS). Immunol Cell Biol 85: 43-45, 2007.

5. Gracias DT and Katsikis PD: MicroRNAs: key components of immune regulation. Adv Exp Med Biol 780: 15-26, 2011.

6. Lieber D and Haas J: Viruses and microRNAs: a toolbox for systematic analysis. Wiley Interdiscip Rev RNA 2: 787-801, 2011

7. Curtale G, Citarella F, Carissimi C, et al: An emerging player in the adaptive immune response: microRNA-146a is a modulator of IL-2 expression and activation-induced cell death in T lymphocytes. Blood 115: 265-273, 2010.

8. Hashimi ST, Fulcher JA, Chang MH, Gov L, Wang S and Lee B: MicroRNA profiling identifies miR-34a and miR-21 and their target genes JAG1 and WNT1 in the coordinate regulation of dendritic cell differentiation. Blood 114: 404-414, 2009.

9. Ceppi M, Pereira PM, Dunand-Sauthier I, et al: MicroRNA-155 modulates the interleukin-1 signaling pathway in activated human monocyte-derived dendritic cells. Proc Natl Acad Sci USA 106: 2735-2740, 2009.

10. Sato F, Tsuchiya S, Meltzer SJ and Shimizu K: MicroRNAs and epigenetics. FEBS J 278: 1598-1609, 2011.

11. Liao $\mathrm{H}, \mathrm{Xu} \mathrm{J}$ and Huang $\mathrm{J}$ : FasL/Fas pathway is involved in dengue virus induced apoptosis of the vascular endothelial cells J Med Virol 82: 1392-1399, 2010.

12. Neseliler S, Narayanan D, Fortis-Santiago Y, Katz DB and Birren SJ: Genetically induced cholinergic hyper-innervation enhances taste learning. Front Syst Neurosci 5: 97, 2011.
13. Luers AJ, Loudig OD and Berman JW: MicroRNAs are expressed and processed by human primary macrophages. Cell Immunol 263: 1-8, 2010.

14. Espada-Murao LA and Morita K: Dengue and soluble mediators of the innate immune system. Trop Med Health 39: 53-62, 2011.

15. Nguyen TH, Nguyen TL, Lei HY, et al: Association between sex, nutritional status, severity of dengue hemorrhagic fever and immune status in infants with dengue hemorrhagic fever. Am J Trop Med Hyg 72: 370-374, 2005.

16. Chakravarti A and Kumaria R: Circulating levels of tumour necrosis factor-alpha and interferon-gamma in patients with dengue and dengue haemorrhagic fever during an outbreak. Indian J Med Res 123: 25-30, 2006.

17. Perez AB, Garcia G, Sierra B, et al: IL-10 levels in Dengue patients: some findings from the exceptional epidemiological conditions in Cuba. J Med Virol 73: 230-234, 2004.

18. Juffrie M, Meer GM, Hack CE, et al: Inflammatory mediators in dengue virus infection in children: interleukin- 6 and its relation to C-reactive protein and secretory phospholipase A2. Am J Trop Med Hyg 65: 70-75, 2001.

19. Dalrymple NA and Mackow ER: Endothelial cells elicit immune-enhancing responses to dengue virus infection. J Virol 86: 6408-6415, 2012.

20. Esteller M: Non-coding RNAs in human disease. Nat Rev Genet 12: 861-874, 2011

21. Guo H, Ingolia NT, Weissman JS and Bartel DP: Mammalian microRNAs predominantly act to decrease target mRNA levels. Nature 466: 835-840, 2010.

22. Kong Y and Han JH: MicroRNA: biological and computational perspective. Genomics Proteomics Bioinformatics 3: 62-72, 2005.

23. Heiss BL, Maximova OA and Pletnev AG: Insertion of microRNA targets into the flavivirus genome alters its highly neurovirulent phenotype. J Virol 85: 1464-1472, 2011.

24. Lee TC, Lin YL, Liao JT, et al: Utilizing liver-specific microRNA-122 to modulate replication of dengue virus replicon. Biochem Biophys Res Commun 396: 596-601, 2010.

25. Pham AM, Langlois RA and TenOever BR: Replication in cells of hematopoietic origin is necessary for Dengue virus dissemination. PLoS Pathog 8: e1002465, 2012.

26. Tolfvenstam T, Lindblom A, Schreiber MJ, et al: Characterization of early host responses in adults with dengue disease. BMC Infect Dis 11: 209, 2011. 